\title{
Fractional quantum Hall effect in graphene
}

\author{
Csaba Tőke, Paul E. Lammert, Vincent H. Crespi, and Jainendra K. Jain \\ Department of Physics, 104 Davey Lab, Pennsylvania State University, University Park PA, 16802
}

(Dated: November 1, 2018)

\begin{abstract}
Unlike regular electron spin, the pseudospin degeneracy of Fermi points in graphene does not couple directly to magnetic field. Therefore, graphene provides a natural vehicle to observe the integral and fractional quantum Hall physics in an elusive limit analogous to zero Zeeman splitting in GaAs systems. This limit can exhibit new integral plateaus arising from interactions, large pseudoskyrmions, fractional sequences, even/odd numerator effects, composite-fermion pseudoskyrmions, and a pseudospin-singlet composite-fermion Fermi sea. The Dirac nature of the $B=0$ spectrum, which induces qualitative changes in the overall spectrum, has no bearing on the fractional quantum Hall effect in the $n=0$ Landau level of graphene. The second Landau level of graphene is predicted to show more robust fractional quantum Hall effect than the second Landau level of GaAs.

PACS numbers: 73.63.Fg, 73.23.-b, 81.05.Uw
\end{abstract}

The discovery of integral quantum Hall plateaus in graphene [1] poses several questions: Does the fractional quantum Hall effect (FQHE) also occur in graphene, and, if so, what is its character? Does it resemble the integral quantum Hall effect (IQHE) in graphene? How does it compare to the FQHE in GaAs? What other physics does interaction produce? Even if the Zeeman energy is large enough to fully polarize the (real) spin of the low energy state, as assumed throughout this article, the graphene Fermi point degeneracy, which takes the form of a pseudospin 1/2 character, remains. Strikingly, despite the qualitative changes in the Landau level (LL) spectrum that arise from the Dirac dispersion of the low energy states at zero field, the physics of the $n=0$ graphene Landau level is identical to that of electrons in the $n=0$ GaAs Landau level, but with the Zeeman splitting set identically to zero; the pseudospin, which is also a sublattice index for $n=0$, plays the role of the traditional electron spin. This leads to the prediction of the formation of composite fermions, FQHE at $\nu=n /(2 p n \pm 1)$, odd versus even numerator effects, and a pseudospin singlet composite fermion Fermi sea. Giant pseudoskyrmions are predicted for the ferromagnetic integral and fractional quantum Hall states. Our numerical diagonalizations show that, interestingly, the FQHE of graphene in the $n=1$ Landau level is more robust than that in the $n=1 \mathrm{GaAs}$ Landau level, due to more favorable interaction pseudopotentials. We assume below that the pseudospin symmetry is exact, and also that LL mixing is negligible.

In graphene, the two Fermi points, each with a twofold band degeneracy, can be described by a low-energy continuum approximation with a four-component envelope wave function whose components are labelled by a Fermi-point pseudospin $= \pm 1$ and a sublattice. The Hamiltonian for one pseudospin component is [2, 3]

$$
H=v_{F}\left(\begin{array}{cc}
0 & \Pi_{x}-i \Pi_{y} \\
\Pi_{x}+i \Pi_{y} & 0
\end{array}\right)
$$

where $v_{F}$ is the Fermi velocity and $\vec{\Pi}=\vec{p}+(e / c) \vec{A}$. Letting $z=x-i y$, choosing the symmetric gauge $\vec{A}=$ $(-B y / 2, B x / 2)$, and introducing the Landau level raising and lowering operators, $a^{\dagger}=\left(\bar{z} / 2-2 \partial_{z}\right) / \sqrt{2}$ and $a=\left(z / 2+2 \partial_{\bar{z}}\right) / \sqrt{2}$, the Hamiltonian becomes

$$
H=\frac{\sqrt{2} \hbar v_{F}}{i l_{B}}\left(\begin{array}{cc}
0 & a \\
-a^{\dagger} & 0
\end{array}\right) .
$$

The eigenvalue problem is conveniently formulated in terms of $H^{2}$, which can be represented as LL number operators:

$$
H^{2}=\frac{2 \hbar^{2} v_{F}^{2}}{l_{B}^{2}}\left(\begin{array}{cc}
a^{\dagger} a+1 & 0 \\
0 & a^{\dagger} a
\end{array}\right) .
$$

Any $\Psi=\left(\begin{array}{c}\alpha \eta_{n-1, m_{1}} \\ \beta \eta_{n, m_{2}}\end{array}\right)$ is an eigenvector of $H^{2}$, where $\eta_{n, m}$ are the standard LL eigenfunctions in GaAs $(n=$ $0,1, \cdots$ is the LL index, and $m$ is the angular momentum index). $H \Psi=E_{n} \Psi$ requires $m_{1}=m_{2}$ and fixes $\beta / \alpha$, with the result that normalized eigenvectors take the form $(\operatorname{sgn}(0)=0$ by convention $)$

$$
\begin{gathered}
\Psi^{(n \neq 0, m)}=\frac{1}{\sqrt{2}}\left(\begin{array}{c}
-\operatorname{sgn}(n) i \eta_{|n|-1, m} \\
\eta_{|n|, m}
\end{array}\right), \\
\Psi^{(0, m)}=\left(\begin{array}{c}
0 \\
\eta_{0, m}
\end{array}\right) \\
E_{n}=\operatorname{sgn}(n) \sqrt{2 \hbar v_{F}^{2} e B|n| / c}
\end{gathered}
$$

Before discussing the FQHE[4], we describe how interactions are expected to affect the integral QHE in graphene. For noninteracting electrons, when both the spin and the pseudospin degeneracies are present, the Hall plateaus have been predicted and seen at

$$
R_{H}=h /\left(j e^{2}\right), j=\ldots,-6,-2,2,6,10,14, \ldots
$$


For magnetic fields large enough to resolve the spin bands the Hall plateaus occur at

$$
R_{H}=h /\left(j e^{2}\right), j=\ldots,-2,-1,0,1,2,4,6,8, \ldots
$$

where $j=0$ implies a band insulator. However, all integral plateaus should, in principle, become possible for interacting electrons. Consider a magnetic field large enough to lift the spin degeneracy at a filling $\nu=2 n+1$ with $n=1,2,3, \ldots$ where no QHE occurs for noninteracting electrons. For interacting electrons, the rotational symmetry in pseudospin space is spontaneously broken due to pseudospin exchange, with the pseudospin magnetization picking an arbitrary direction. While this system supports gapless pseudospin-wave excitation, the charged excitations have a gap, thus producing a Hall plateau.

FQHE in the $n=0$ Landau level of graphene: The unusual electronic dispersion of graphene around $E=0$ is reflected in both the structure of Landau levels and in the offset of the Hall conductance staircase. Nonetheless, the actual wave functions of electrons in the $n=0$ manifold are identical to those in the conventional lowest Landau level of GaAs. Consequently, the FQHE in the $n=0$ Landau level of graphene at a high magnetic field maps onto FQHE of electrons in GaAs with zero Zeeman energy [5], as corroborated and extended by the numerical results described below.

The basic physics in the $n=0$ graphene LL, therefore, is the same as in GaAs. Each electron captures an even number $(2 p)$ of quantized vortices to become a composite fermion [6]. The Berry phases generated by the vortices effectively cancel part of the external magnetic field, so that the dynamics of composite fermions are governed by a reduced magnetic field $B^{*}=B-2 p \rho \phi_{0}$, where $\rho$ is the particle density in the $n=0$ Landau level and $\phi_{0}=h c / e$ is the flux quantum. The $n=0$ Landau level of electrons splits into Landau-like levels of composite fermions, whose filling factor $\nu^{*}$, in terms of the electron filling $\nu$, is given by $\nu=\nu^{*} /\left(2 p \nu^{*} \pm 1\right)$.

The IQHE of composite fermions for $\nu^{*}=n$ produces sequences of fractions [7]:

$$
\nu=n /(2 p n \pm 1) .
$$

The origin of gap, i.e. the energy required to promote a composite fermion into a higher CF-Landau level, is different for even and odd values of $n$. For even $n$, the ground state is a pseudospin singlet, with $n / 2 \mathrm{CF}$-Landau levels for each component of the pseudospin occupied. For odd $n$, the ground state is partially pseudospin polarized; no QHE would occur here if the composite fermions did not interact, but the residual interaction between composite fermions opens a gap. To the extent the residual interaction is weak, one expects fractions with even numerators to be more robust than those with odd numerators. The excitation energies for the GaAs FQHE in the zero Zeeman energy limit apply to graphene FQHE within the $n=0$ level. Calculated gaps to creation of a far-separated charged quasiparticle/quasihole pair at $\nu=1 / 3$ and (unpolarized) $\nu=2 / 5$ are 0.07 and 0.04 $e^{2} / \epsilon \ell_{B}$, respectively [8]; the larger gap at $1 / 3$ indicates the significance of inter-CF interactions.

So long as electrons are confined to the $n=0$ Landau level, they have no memory of the Dirac nature of the zero-field dispersion, with some surprising consequences for the FQHE. The CF-cyclotron energy opens up approximately linearly with $B^{*}$, as expected for composite fermions with a parabolic dispersion, even though the cyclotron energy of electrons in graphene scales anomalously with $B$.

In GaAs, the sequence of FQHE states at $\nu=n /(2 p n \pm$ 1) terminates as $n \rightarrow \infty$ in a composite-fermion Fermi sea at $\nu=1 / 2 p$, where the effective magnetic field vanishes 9, 10]. For zero Zeeman energy, variational calculations favor the spin singlet Fermi sea 11], so graphene should have a pseudospin-singlet CF Fermi sea at $\nu=1 / 2 p$. The CF Fermi sea in GaAs has been successfully modeled as an ordinary Fermi sea with parabolic dispersion, which allows one to deduce an effective mass for composite fermions. The same should be true of the CF Fermi sea in graphene, in spite of the fact that electrons in graphene make a Dirac sea at zero magnetic field and have no effective mass. The singlet nature of the CF Fermi sea can be ascertained through a measurement of the Fermi wave vector, as was accomplished in GaAs sys-

\begin{tabular}{|r|r|r|r|r|c|c|c|c|c|c|c|}
\hline \hline$\nu$ & $N$ & $2 Q$ & $D$ & $D_{0}$ & $L^{(0)}$ & $S^{(0)}$ & \multicolumn{2}{|c|}{ GaAs } & \multicolumn{2}{|c|}{ graphene } & overlap \\
\cline { 6 - 10 } & & & & & & $L^{(1)}$ & $S^{(1)}$ & $L^{(1)}$ & $S^{(1)}$ & \\
\hline $1 / 3$ & 4 & 9 & 145 & 5 & 0 & 2 & 0 & 2 & 0 & 2 & 0.99932 \\
& 5 & 12 & 1106 & 10 & 0 & $5 / 2$ & 2 & $3 / 2$ & 0 & $5 / 2$ & 0.99998 \\
& 6 & 15 & 11588 & 50 & 0 & 3 & 0 & 3 & 0 & 3 & 0.99875 \\
& 7 & 18 & 109138 & 290 & 0 & $7 / 2$ & 0 & $7 / 2$ & 0 & $7 / 2$ & 0.99974 \\
\hline $2 / 3$ & 4 & 5 & 29 & 3 & 0 & 0 & 0 & 2 & 0 & 0 & 0.99968 \\
& 6 & 8 & 500 & 10 & 0 & 0 & 1 & 3 & 0 & 0 & 0.98887 \\
& 8 & 11 & 11483 & 91 & 0 & 0 & 2 & 4 & 0 & 0 & 0.97482 \\
\hline $2 / 5$ & 4 & 7 & 72 & 4 & 0 & 0 & 0 & 0 & 0 & 0 & 0.99248 \\
& 6 & 12 & 3796 & 28 & 0 & 0 & 1 & 3 & 0 & 0 & 0.93622 \\
& 8 & 17 & 274842 & 768 & 0 & 0 & 0 & 4 & 0 & 0 & 0.95578 \\
\hline $3 / 5$ & 5 & 8 & 226 & 5 & 0 & $3 / 2$ & 0 & $5 / 2$ & 0 & $3 / 2$ & 1.00000 \\
& 8 & 13 & 39131 & 205 & 0 & 2 & 0 & 4 & 0 & 0 & 0 \\
\hline \hline
\end{tabular}

TABLE I: Orbital angular momentum $L^{(n)}$ and spin/pseudospin $S^{(n)}$ of the ground states of finite systems on a sphere at $\nu=\frac{1}{3}, \frac{2}{3}, \frac{2}{5}$ and $\frac{3}{5}$ in the $n=0$ and $n=1$ Landau levels of graphene and GaAs. $D$ is the dimension of the Hilbert space in the $L_{z}=S_{z}=0$ sector; $D_{0}$ is the dimension of the $L=0$ sector. The last column gives the overlaps between the $n=0$ and $n=1$ graphene ground states. 
tems by various geometric means [10].

FQHE in the $n=1$ Landau level of graphene: The nature of FQHE depends on the Haldane pseudopotentials. In GaAs, the FQHE is essentially restricted to the lowest LL: very few fractions are seen in $n=1$, and almost none in higher LLs. The mapping between GaAs and graphene does not hold in higher Landau levels, so different behaviors are expected. We first evaluate the Coulomb matrix elements within the $n$th graphene Landau level. Write $\left|n_{1}, m_{1} ; n_{2}, m_{2} ; \ldots ; n_{N}, m_{N}\right\rangle$ for the product state $\eta_{n_{1}, m_{1}} \otimes \eta_{n_{2}, m_{2}} \otimes \cdots \otimes \eta_{n_{N}, m_{N}}$ and $\left.\left.\| n_{1}, m_{1} ; n_{2}, m_{2} ; \ldots ; n_{N}, m_{N}\right\rangle\right)$ for $\Psi^{\left(n_{1}, m_{1}\right)} \otimes \cdots \otimes$ $\Psi^{\left(n_{N}, m_{N}\right)}$. Then,

$$
\begin{aligned}
& 4\left\langle\left\langle n, m_{1} ; n, m_{2}\|V\| n, m_{3} ; n, m_{4}\right\rangle\right\rangle= \\
& =\left\langle n, m_{1} ; n, m_{2}|V| n, m_{3} ; n, m_{4}\right\rangle+ \\
& \left\langle n-1, m_{1} ; n, m_{2}|V| n-1, m_{3} ; n, m_{4}\right\rangle+ \\
& \left\langle n, m_{1} ; n-1, m_{2}|V| n, m_{3} ; n-1, m_{4}\right\rangle+ \\
& \left\langle n-1, m_{1} ; n-1, m_{2}|V| n-1, m_{3} ; n-1, m_{4}\right\rangle .
\end{aligned}
$$

By conservation of angular momentum, these matrix elements are all proportional to $\delta_{m 1+m 2, m 3+m 4}$. The problem of electrons in the $n^{\text {th }}$ graphene LL thus formally maps into that of the lowest GaAs LL (with two pseudospin copies) with an effective interaction defined by the pseudopotentials

$$
\begin{gathered}
V_{m}^{(n) \text { graphene }}=\frac{1}{4}\left[V_{m}^{(n)}+V_{m}^{(n-1)}+2 V_{m}^{(n, n-1)}\right] \\
V_{m}^{(n, n-1)}=\int \frac{d^{2} k}{(2 \pi)^{2}} \frac{2 \pi}{k} L_{n}\left(\frac{k^{2}}{2}\right) L_{n-1}\left(\frac{k^{2}}{2}\right) e^{-k^{2}} L_{m}\left(k^{2}\right),
\end{gathered}
$$

and $V_{m}^{(n)}$ is the effective pseudopotential for the $n^{\text {th }}$ LL

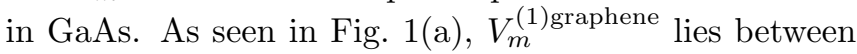
$V_{m}^{(0)}$ and $V_{m}^{(1)}$, except for $m=1$. To see what FQHE this interaction implies, we have numerically diagonalized finite systems in a geometry where $N$ electrons move on the surface of a sphere whose center holds a magnetic monopole of strength $Q$ producing a flux of $2 Q \mathrm{hc} / \mathrm{e}$ through the surface of the sphere. We use the pseudopotentials calculated above for the disk geometry; this gives the exact result for very large systems and is generally a reasonable approximation.

As a preliminary step, we have investigated the fully pseudospin-polarized sector, where larger systems (with up to ten particles) can be studied, and found that the $n=1$ graphene LL behaves very similarly to the $n=0$ LL. This suggests that the FQHE in higher graphene LLs may be more robust than in GaAs. We next compare ground states for graphene systems including the pseudospin degree of freedom (but with the real spin frozen), to GaAs systems with zero Zeeman splitting. Table \ shows the ground state quantum numbers, orbital angular momentum $L$ and spin or pseudospin $S$, for the $n=0$ and $n=1$ LLs at several fractions. The $n=0$ results are identical for GaAs and graphene. The near perfect overlaps in the last column of the table indicate that the FQHE in the $n=1$ graphene LL also strongly resembles that in the $n=0$ (lowest) graphene LL. (The analogous overlaps are rather low for the FQHE states in the $n=0$ and $n=1 \mathrm{LLs}$ in GaAs 12].) The $1 / 3$ state is fully polarized, whereas the $2 / 5$ and $2 / 3$ are (pseudo)spin singlet. At $3 / 5$ the spin of the ground state differs from that of the lowest LL for $N=8$; the existence or the nature of FQHE at this fraction remains unclear at the moment.

Pseudoskyrmions: In GaAs quantum wells, the excitations of the $\nu=1$ state for exactly zero Zeeman splitting are not simple particle-hole excitations but spin textures called skyrmions [13, 14, 15], in which half of the spins are reversed. However, the skyrmion size rapidly decreases with increasing Zeeman energy; experimentally, skyrmions typically have 3 to 5 flipped spins [16, 17]. No skyrmions occur at $\nu=3,5, \ldots$. CF skyrmions are believed to be relevant near $\nu=1 / 3$ at very small Zeeman energies [18, 19, 20].

In contrast, for the state in which one of the two degenerate levels of the $n=0$ graphene manifold is fully occupied (which produces zero Hall conductance), the excitations ought to be large pseudoskyrmions. In exact diagonalization studies, we find pseudoskyrmions also in the $n=1,2$ graphene LLs, where the addition of one particle or hole to the fully pseudospin polarized state produces a pseudospin singlet state. No such behavior is seen for $n \geq 3$; here the excitation is fully pseudospin-polarized $(S=N / 2)$ on the quasihole side and has a single pseudospin reversed $(S=N / 2-1)$ on the quasiparticle side. Fig. 1(b) depicts the $N$ dependence of the gap to creating a pair of pseudoskyrmion and anti-pseudoskyrmion, computed by exact diagonalization. (We follow the convention of Ref. [14] to define the pseudoskyrmion gap in terms of "neutral" quasiparticle/quasihole energies [21].) Extrapolation to the thermodynamic limit yields $\Delta_{1}^{(0)}=$ $0.606(15), \Delta_{1}^{(1)}=0.126(7)$, and $\Delta_{1}^{(2)}=0.18(1)$. The gap in the $n=0 \mathrm{LL}$ is consistent with $\sqrt{\pi / 8}\left(e^{2} / \epsilon \ell_{B}\right)$, half the energy required to create an ordinary particle-hole pair excitation [14]. We note that the pseudospin texture in the $n=0$ graphene LL can be imaged directly by scanning tunneling microscopy, since an electron's pseudospin determines on which sublattice it resides.

Fig. 1( c-d) shows the gaps as a function of the number of particles for several fractional filling factors in the $n$th graphene LL. The lowest-energy charged excitations at $\nu^{(1)}=1 / 3$ are $\mathrm{CF}$ pseudoskyrmions (pseudo-spin singlet state), with an excitation energy of 0.017 , to be compared to 0.096 for a (pseudospin reversed) particle-hole pair of composite fermions. (The latter gap is greater than in the $n=0$ LL because the $V_{1}$ pseudopotential is greater in the $n=1$ than in the $n=0 \mathrm{LL}$.) The gaps at filling factors $2 / 5$ and $2 / 3$ involve pseudospin reversal for composite 

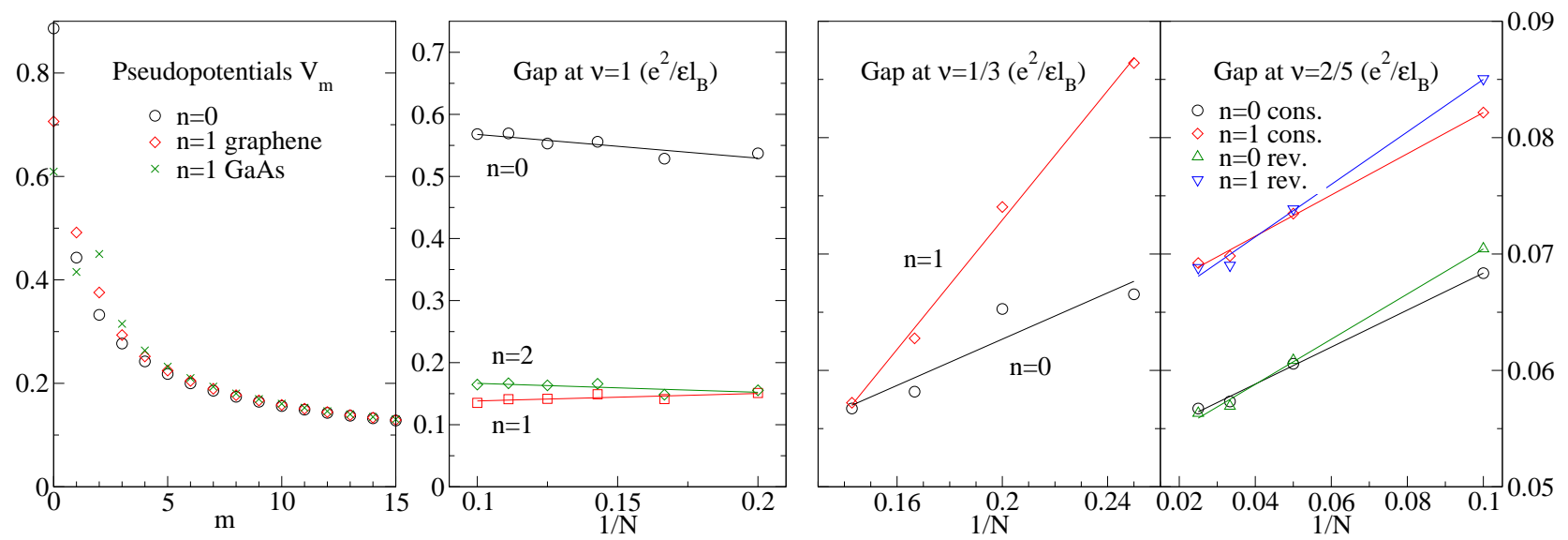

FIG. 1: (a) Pseudopotentials for $n=0$ and $n=1$ Landau levels in graphene and GaAs. (b)-(d) Activation gaps at $\nu^{(n)}=1,1 / 3$ and $2 / 5$ in several graphene LLs. All energies are given in units of $e^{2} / \epsilon l_{B}$, where $\epsilon$ is the dielectric constant of the host semiconductor and $l_{B}$ is the magnetic length. The gaps in (b) and (c) refer to the energy required to create a pseudoskyrmion-antiskyrmion pair. Gaps to pseudospin conserving (cons.) and pseudospin reversed (rev.) excitations are given in $(\mathrm{d})$.

fermions. For $2 / 5$ the gaps, $\Delta_{2 / 5}^{(0)}=0.051(1)$ and $\Delta_{2 / 5}^{(1)}=$ $0.062(1)$, were obtained from the trial wave functions of the CF theory, evaluated by a procedure described in Ref. 22 .

We summarize our principal conclusions. The lowest LL FQHE of graphene in the large Zeeman energy limit is equivalent to the lowest LL FQHE in GaAs in the zero Zeeman energy limit, terminating into a pseudospinsinglet Fermi sea at half filling. The effective interaction in Eq. (11) is shown to be more favorable to CF formation in the $|n|=1 \mathrm{LL}$ of graphene than in the $n=1 \mathrm{LL}$ of GaAs. The gaps at $\nu^{(1)}=1 / 3,2 / 5$ are calculated and FQHE is predicted at $\nu^{(1)}=2 / 3$ due to reverse flux attachment. In contrast to GaAs, skyrmions are predicted to occur at $\nu^{(n)}=1,3$ in the $n=0,1,2$ LLs.

While completing the manuscript, we learned from Philip Kim of the observation of new integral plateaus by Zhang et al. 23]. Several recent theory preprints have examined similar topics, including a field theory approach that obtains results similar to ours 24] and derivations of the Coulomb pseudopotentials for graphene 25]. Support by the National Science Foundation under grants no. DMR-0240458, DMR-0305035 and ECS-0609243 is gratefully acknowledged.

[1] K.S. Novoselov et al., Nature 438, 197 (2005); Y. Zhang et al., Nature 438, 201 (2005);

[2] N. H. Shon and T. Ando, J. Phys. Soc. Jpn. 67, 2421 (1998).

[3] G.W. Semenoff, Phys. Rev. Lett. 53, 2449 (1984).

[4] D.C. Tsui, H.L. Stormer, and A.C. Gossard, Phys. Rev. Lett. 48, 1559 (1982).

[5] X.G. Wu, G. Dev, and J.K. Jain, Phys. Rev. Lett. 71,
153 (1993); K. Park and J.K. Jain, ibid. 80, 4237 (1998). [6] J.K. Jain, Phys. Rev. Lett. 63, 199 (1989).

[7] The index $n$ is used for both electron and CF Landau levels; the meaning should be clear from the context. Also, $\nu^{(n)}$ denotes the filling factor in the $n$th LL, with the lower LLs assumed to be filled and inert.

[8] E.H. Rezayi, Phys. Rev. B 36, 5454 (1987); S.S. Mandal and J.K. Jain, ibid. 63, 201310 (2001).

[9] V. Kalmeyer and S.C. Zhang, Phys. Rev. B 46, R9889 (1992); B.I. Halperin, P.A. Lee, and N. Read, Phys. Rev. B 47, 7312 (1993).

[10] R.L. Willett et al., Phys. Rev. Lett. 71, 3846 (1993); W. Kang et al., ibid. 71, 3850 (1993); V.J. Goldman et al., ibid. 72, 2065 (1994); J. H. Smet et al., ibid. 77, 2272 (1996).

[11] K. Park et al., Phys. Rev. B 58, R10167 (1998); K. Park and J.K. Jain, Phys. Rev. Lett. 80, 4237 (1998).

[12] N. d'Ambrumenil and A.M. Reynolds, J. Phys. C: Solid State Phys. 21, 119 (1988).

[13] D.-H. Lee and C. L. Kane Phys. Rev. Lett. 64, 1313 (1990);

[14] S.L. Sondhi et al., Phys. Rev. B 47, 16419 (1993).

[15] K. Moon et al., Phys. Rev. B 51, 5138 (1995).

[16] H.A. Fertig et al., Phys. Rev. B 50, R11018 (1994).

[17] S. E. Barrett et al., Phys. Rev. Lett. 74, 5112 (1995); A. Schmeller et al., ibid. 75, 4290 (1995); E. H. Aifer, B. B. Goldberg, and D. A. Broido, ibid. 76, 680 (1996).

[18] R.K. Kamilla, X.G. Wu, and J.K. Jain, Phys. Rev. Lett. 76, 1332 (1996).

[19] A. Wójs and J. J. Quinn, Phys. Rev. B 66, 045323 (2002).

[20] D. R. Leadley et al., Phys. Rev. Lett. 79, 4246 (1997).

[21] A. H. MacDonald and S. M. Girvin, Phys. Rev. B 34, 5639 (1986).

[22] C. Tőke et al., Phys. Rev. B 72, 125315 (2005).

[23] Y. Zhang et al., Phys. Rev. Lett. 96, 136806 (2006).

[24] K. Yang, S. Das Sarma, and A.H. MacDonald, cond-mat/0605666 (2006).

[25] K. Nomura and A. H. MacDonald, Phys. Rev. Lett. 96, 256602 (2006); V. .M. Apalkov and T. Chakraborty, 
cond-mat/0606037 (2006); M. O. Goerbig, R. Moessner, and B. Douçot, cond-mat/0604554 (2006). 